\title{
Causality in Quantum Field Theory with Nonlocal Interaction
}

\author{
V. A. Alebastrov and G. V. Efimov \\ Joint Institute for Nuclear Research, Laboratory of Theoretical Physics, Moscow, USSR
}

Received March 1, 1974

\begin{abstract}
It is proved that the $S$-matrix satisfies the Bogolubov microcausality condition in each order in perturbation theory in a quantum field theory with nonlocal interaction, where the nonlocality is introduced with the help of form factors being entire analytical functions of the order $1 / 2$.
\end{abstract}

\section{Introduction}

It is well known that the principle of causality is the basis of all approaches in the theory of elementary particles. In quantum field theory this principle, like the postulates of relativistic covariance and quantum nature of phenomena in the microworld, is of fundamental importance (construction of the $S$-matrix [1], the Wightman axiomatic approach [2], dispersion relations [3] and so on).

In local quantum field theory causality is manifested as a requirement for the Heisenberg fields $\phi(x)$ to be locally commutable

$$
[\phi(x), \phi(y)]_{-}=0 \quad \text { for } \quad x \sim y
$$

or as the microcausality condition for the $S$-matrix:

$$
\frac{\delta}{\delta \phi(x)}\left(\frac{\delta S}{\delta \phi(y)} S^{+}\right)=0 \text { for } x \lesssim y .
$$

The both conditions are of a formal mathematical nature and are the postulates describing the mathematical structure of the fields $\phi(x)$ in (1.1) and of the $S$-matrix in (1.2) rather than requirements of physical causality. This is explained by the fact that the concept of point-like nature of events suggested in (1.1) and (1.2) is incompatible with the ideas of relativistic quantum mechanics.

Over the last time numerous attempts have been made to formulate the so-called physical condition of causality [4], i.e. to find minimal requirements on the amplitudes of physical processes which would guarantee the absence of any obviously noncausal phenomena in the macroworld. However, this problem remains still open and the conditions 
(1.1) and (1.2) are actually the only working formulations of causality ensuring the well-known results in different approaches of local quantum field theory.

It should be noted that the predictions of the local theory are in satisfactory agreement with experiment up to the presently reachable distances $\left(\approx 10^{-15} \mathrm{~cm}\right)$. This appears to indicate that, firstly, the mathematical condition of causality guarantees undoubtedly the fulfilment of the physical condition if the latter will successfully be formulated. Secondly, the notion of point-like nature of events is a good approximation up to presently reachable energies.

However, troubles due to ultraviolet divergences as well as the fact that the notion of strict localizability of events is only approximate, stimulated many attempts to formulate a selfconsistent theory at the expense of locality and, which seems to be quite obvious, at the expense of microcausality. However, in so doing, we may consider the theory as valid only if the violation of microcausality is localized in sufficiently small space-time regions. The investigation of the causal $S$-matrix properties in this kind of theories is of predominant importance.

In the present paper we show that the quantum field theory with nonlocal interaction proposed by one of the authors [5] satisfies the microcausality condition (1.2) in each perturbation order.

\section{The Lagrangian and the $S$-Matrix}

We consider the theory of an one-component scalar field with the Lagrangian:

$$
\begin{aligned}
\mathscr{L}(x) & =\mathscr{L}_{0}(x)+g \mathscr{L}_{I}(x), \\
\mathscr{L}_{0}(x) & =\frac{1}{2}:\left[\partial_{\mu} \varphi(x) \partial_{\mu} \varphi(x)-m^{2} \varphi^{2}(x)\right]:, \\
g \mathscr{L}_{I}(x) & =g:[\phi(x)]^{N}: .
\end{aligned}
$$

Here $N$ is an integer,

where

$$
\phi(x)=\int d x^{\prime} K\left(x-x^{\prime}\right) \varphi\left(x^{\prime}\right)=K\left(l^{2} \square\right) \varphi(x)
$$

$$
K\left(x-x^{\prime}\right)=K\left(l^{2} \square\right) \delta^{(4)}\left(x-x^{\prime}\right)
$$

is a nonlocal distribution (see [6]). The parameter $l$ implies the dimension of the interaction region.

Generally speaking, we may consider a more general case (nonpolynomial Lagrangians and different methods of introducing nonlocality), as we did when proving the unitarity of the $S$-matrix in [7]. However we are not going to do this here in order not to make our presentation cumbersome. The methods proposed below can easily be applies to the general case. 
The introduction of nonlocality into the interaction Lagrangian in this way leads to the fact that in the perturbation series for the $S$-matrix the propagator of our scalar particle changes in the following way:

$$
\frac{1}{m^{2}-k^{2}-i \varepsilon} \rightarrow \frac{\left[K\left(l^{2} k^{2}\right)\right]^{2}}{m^{2}-k^{2}-i \varepsilon}
$$

We suppose that the function $V(z)=[K(-z)]^{2}$ satisfies the properties $(E)$ :

1) $V(z)$ is an entire analytical function of the order $\varrho=\frac{1}{2}$, i.e. $\exists C>0$, $l_{1}>0$ so that

$$
|V(z)| \leqq C \exp \left\{l_{1} \sqrt{|z|}\right\} ;
$$

2) $V(z)=0\left(|z|^{-1-a}\right)$ when $\operatorname{Re} z \rightarrow+\infty(0<a<1)$;

3) $V\left(-l^{2} m^{2}\right)=1$

4) $[V(z)]^{*}=V\left(z^{*}\right)$.

For example,

$$
V(z)=\left(\frac{\sin l \sqrt{m^{2}-k^{2}}}{l \sqrt{m^{2}-k^{2}}}\right)^{4} \quad\left(z=-l^{2} k^{2}\right) .
$$

The following representation is valid for the functions satisfying the conditions

$$
V\left(-l^{2} k^{2}\right)=\int_{Q^{2} \leqq l^{2}} d^{4} \varrho a\left(\varrho^{2}\right) e^{k_{0} \varrho_{0}+i k \varrho}
$$

where the integration is performed over the Euclidean globe

$$
\varrho^{2}=\varrho_{0}^{2}+\varrho_{1}^{2}+\varrho_{2}^{2}+\varrho_{3}^{2}=\varrho_{0}^{2}+\varrho^{2} \leqq l^{2} .
$$

The function $a\left(\varrho^{2}\right)$ is supposed to be integrable for $\varrho^{2} \leqq l^{2}$.

The space of the test functions $Z$ consists of all entire functions of an arbitrary order $f\left(z_{1}, \ldots, z_{n}\right)$ of $n$ complex variables $z_{j}=x_{j}+i y_{j}(j=1,2, \ldots, n)$ such that

$$
\int_{-\infty}^{\infty} d x_{1} \ldots \int_{-\infty}^{\infty} d x_{n}\left|f\left(x_{1}+i y_{1}, \ldots, x_{n}+i y_{n}\right)\right|<\infty
$$

for any $y_{1}, \ldots, y_{n}$.

The space $\tilde{Z}$, which is the space of the Fourier transforms of $f \in Z$, consists of the differentiable functions $\tilde{f}\left(p_{1}, \ldots, p_{n}\right)$ which satisfy the condition: there always exists positive $C_{\left\{N_{j}\right\}}$, such that

for any positive $N_{1}, \ldots, N_{n}$.

$$
\left|\tilde{f}\left(p_{1}, \ldots, p_{n}\right)\right| \leqq C_{\left\{N_{j j}\right\}} \exp \left\{-\sum_{j=1}^{n} N_{j}\left|p_{j}\right|\right\}
$$

Let us note that $D \bar{C} Z$ but $D \subset \tilde{Z}$, where $D$ is the space of the infinitely differentiable functions with a finite support. We define the variational 
derivative of a functional

$$
F[f]=\tilde{F}[\tilde{f}] \quad(f \in Z, \tilde{f} \in \tilde{Z})
$$

over $f(x) \in Z$ as

$$
\frac{\delta}{\delta f(x)} F[f] \underset{\mathrm{Df}}{=} \int d p e^{-i p x} \frac{\delta}{\delta \tilde{f}(p)} \tilde{F}[\tilde{f}] .
$$

Making use of the standard methods of quantum field theory [1] one obtains that the $S$-matrix is formally representable as

$$
\begin{aligned}
S[g] & =T \exp \left\{i \int d x g(x) \mathscr{L}_{I}(x)\right\} \\
& =\sum_{n=0}^{\infty} \frac{i^{n}}{n !} \int d x_{1} \ldots \int d x_{n} g\left(x_{1}\right) \ldots g\left(x_{n}\right) S_{n}\left(x_{1}, \ldots, x_{n}\right) .
\end{aligned}
$$

Here $g(x)$ is the interaction switching on function. The operator $S_{n}\left(x_{1}, \ldots, x_{n}\right)$ can be expanded in the normal products of the quantized field $\varphi(x)$ :

$$
S_{n}\left(x_{1}, \ldots, x_{n}\right)=\sum_{m_{1} \ldots m_{n}} K_{m_{1} \ldots m_{n}}^{(n)}\left(x_{1}, \ldots, x_{n}\right): \frac{\varphi^{m_{1}}\left(x_{1}\right)}{m_{1} !} \ldots \frac{\varphi^{m_{n}}\left(x_{n}\right)}{m_{n} !}:
$$

The coefficient functions $K_{m_{1} \ldots m_{n}}^{(n)}\left(x_{1}, \ldots, x_{n}\right)$ are expressed in terms of the products of the nonlocal propagators of the field $\varphi(x)$ :

$$
D\left(x_{1}-x_{2}\right)=\frac{1}{(2 \pi)^{4} i} \int \frac{d^{4} k V\left(-l^{2} k^{2}\right) e^{-i k\left(x_{1}-x_{2}\right)}}{m^{2}-k^{2}-i \varepsilon} .
$$

As far as in virtue of the conditions $(E)$ this function exists as a distribution on $Z$ the products of the nonlocal propagators are not defined mathematically. The definition of the products of the type

$$
K_{m_{1} \ldots m_{n}}^{(n)}\left(x_{1}, \ldots, x_{n}\right)=\prod_{s, t} D\left(x_{s}-x_{t}\right)
$$

is the main problem to be solved in the construction of the finite $S$-matrix.

The coefficient functions $K_{m_{1} \ldots m_{n}}^{(n)}\left(x_{1}, \ldots, x_{n}\right)$ are constructed as a limit of the locally integrable functions $K_{m_{1} . . m_{n}}^{(n) \delta}\left(x_{1}, \ldots, x_{n}\right)$ by means of an intermediate regularization procedure given by the parameter $\delta$, so that in the improper sense there exists a limit

$$
\lim _{\delta \rightarrow 0} K_{m_{1} \ldots m_{n}}^{(n) \delta}\left(x_{1}, \ldots, x_{n}\right)=K_{m_{1} \ldots m_{n}}^{(n)}\left(x_{1}, \ldots, x_{n}\right) \in Z^{\prime}
$$

or, otherwise,

where $g(x) \in Z$.

$$
S[g]=\lim _{\delta \rightarrow 0} S^{\delta}[g]
$$

In paper [7] the regularization procedure is formulated in a such way that the limit (2.9) exists and the $S[g]$-matrix is unitary in each order of perturbation theory. 
A somewhat other regularization which allows performing canonical quantization of the nonlocal Lagrangian (2.1) and possesses the same properties is formulated in [8].

\section{The Condition of Causality}

In what follows we verify the following condition of causality

$$
R(x, y)=\frac{\delta}{\delta g(x)}\left(\frac{\delta S[g]}{\delta g(y)} S^{+}[g]\right)=0
$$

when $x \lesssim y$. Let us put $y=0$, then we have to prove that for $M(x)$ $=\langle\alpha|R(x, 0)| \beta\rangle(|\alpha\rangle$ and $|\beta\rangle$ are arbitrary physical states) the integral

$$
\int d^{4} x M(x) f_{G}(x)=0
$$

for any functions $f_{G}(x)$ which differs from zero only in space-time regions $G \subset R^{4}$ lying outside the future cone:

$$
G \bar{C} V^{+}=\left\{x: x_{0} \geqq 0, x^{2} \geqq 0\right\} .
$$

Thus, in order to verify the causality condition (3.2) it is enough to have in the space of test functions on which the theory under consideration is constructed, some subspaces of the functions with finite supports. Moreover, the fact of the presence of the functions with finite supports in the space of test functions was taken as a principle of strict locality in quantum field theory (see $[9,10]$ ).

The main trouble of formulating and verifying the causality condition in nonlocal quantum field theory is the fact that the functions with finite supports are absent in the space of the test functions $Z$. Therefore the causality condition formulated above cannot be verified directly. Thus the problem we encounter in investigating the causal properties of the $S$-matrix in our nonlocal theory is to find a method which would permit studying the space-time properties of the distributions defined on the spaces of analytical functions.

We shall consider that the space-time properties of the distributions defined on $Z$ can be determined with the help of projecting sequences of functions $\left\{f_{G, \lambda}(x)\right\}$. The main properties of these sequences are the following:

1.

$$
\begin{gathered}
f_{G, \lambda}(x+i y) \in Z, \quad \lambda>0, \\
\lim _{\lambda \rightarrow 0} f_{G, \lambda}(x+i y)=\left\{\begin{array}{l}
0, x \bar{\epsilon} G, \forall y, \\
\psi(x+i y) \neq 0, x \in G, \forall y .
\end{array}\right.
\end{gathered}
$$

2.

A detailed consideration of the properties of the projecting sequences will be given below (see also [11]). 
We suggest that the $S$-matrix satisfies the microcausality condition if

$$
\lim _{\lambda \rightarrow 0} \int d^{4} x M(x) f_{G, \lambda}(x)=0
$$

for any projecting sequences $\left\{f_{G, \lambda}(x)\right\}$ for which the region $G$ satisfies the condition (3.3).

It is easy to see that in the case of localizable theories the definition (3.4) coincides with the usual formulation of the microcausality.

\section{The Projecting Sequences of Functions}

The projecting sequences of functions are defined in the following mainer (see [11]).

Definition. Let 3 be a space of all entire functions $f\left(z_{0}, z_{1}, z_{2}, z_{3}\right)$ of four complex variables in $C^{4}=R^{4}+i R^{4}$. The sequence of functions $\left\{f_{\Gamma, \lambda}(x)\right\}$ is called projecting, if

1. the region $\Gamma \subset R^{4}$;

2. $f_{\Gamma, \lambda}(z)=f_{\Gamma, \lambda}\left(z_{0}, z_{1}, z_{2}, z_{3}\right) \in 3, \lambda>0$;

3. $\int d^{4} x f_{\Gamma, \lambda}(x+i y)=$ const $\Gamma, \lambda>0, \forall y$;

4. $\left|f_{\Gamma, \lambda}(x+i y)\right|=O\left(\frac{1}{\|y\|}\right),\|y\| \rightarrow \infty, x \bar{\epsilon} \Gamma$;

5. $\lim _{\lambda \rightarrow 0} f_{\Gamma, \lambda}(x+i y)=0, x \bar{\in} \Gamma, \forall y$;

6. $\lim _{\lambda \rightarrow 0} f_{\Gamma, \lambda}(x+i y)=\psi(x+i y), x \in \Gamma \subset R^{4}, \forall y$.

$\psi(z)$ is analytic in $\bar{\Gamma}+\mathrm{i} R^{4}$.

The region $\Gamma \subset R^{4}$ is called the support of the projecting sequence.

Let us denote the space of all projecting sequences for any arbitrary support $\Gamma$ by $\Pi$ and the space of the projecting sequences, the supports of which are contained in a region $G \subset R^{4}$, by $\Pi(G)$.

It is essential to note that the functions belonging to $\Pi$ have an infinite order of growth, i.e.

$$
\lim _{r \rightarrow \infty} \frac{r^{N}}{\max _{|| z||=r} \log ^{+}\left|f_{\Gamma, \lambda}(z)\right|}=0, \quad \forall N>0
$$

if $f_{\Gamma, \lambda} \in \Pi$. Therefore

$$
f_{\Gamma, \lambda}(z) \in Z \text {. }
$$

The space $\Pi$ is not empty. The functions of this space can be constructed in the following way, for example. In $Z \subset 3$ there exists functions $g(z)$ of one complex variable which satisfy the following conditions: 
1. There exist a number $d>0$ depending on $g(z)$ such that

$$
|g(z)|=O\left(\frac{1}{|z|}\right)
$$

when $z \rightarrow \infty$ outside the region $|\operatorname{Re} z| \leqq d$.

2. $\int_{-\infty}^{\infty} d x g(x)=1$.

We give an example of such a function

$$
g(z)=\frac{1}{2 \pi} \int_{-\infty}^{\infty} \frac{d u e^{i u z}}{\Gamma\left(\sqrt{1+u^{2}}\right)}=\frac{1}{\pi} \int_{0}^{\infty} \frac{d u \operatorname{Cos} z u}{\Gamma\left(\sqrt{1+u^{2}}\right)} .
$$

The following estimates are valid for this function

$$
g(z)= \begin{cases}O\left(e^{-|x|}\right),|\operatorname{Re} z|>\frac{\pi}{2}, & z \rightarrow \infty \\ O\left(\exp \left\{e^{|z|}\right\}\right),|\operatorname{Re} z|<\frac{\pi}{2}, & z \rightarrow \infty\end{cases}
$$

Let us construct a sequence of functions:

$$
g_{\Gamma, \lambda}(z)=\int_{\Gamma_{\lambda}} d^{4} x^{\prime} \psi\left(x^{\prime}\right) \prod_{j=0}^{3} \frac{1}{\lambda} g\left(\frac{z_{j}-x_{j}^{\prime}}{\lambda}\right) .
$$

Here $\psi(x)$ is an arbitrary function which is analytic in $\left(\bar{\Gamma}+i R^{4}\right)$. For example, $\psi(x)=e^{i q x}$ where $q$ is a four-vector,

$$
\Gamma_{\lambda}=\left\{x: \min _{\xi \in S}\|x-\xi\| \geqq \lambda d, x \in \Gamma, \xi \in S=\bar{\Gamma} \backslash \Gamma\right\},
$$

$\Gamma$ is a bounded region in $R^{4}$.

The sequence (4.3) possesses the following properties.

$$
\lim _{\lambda \rightarrow 0} g_{\Gamma, \lambda}(z)=\psi(z) \Theta_{\Gamma}(x)
$$

where

$$
\Theta_{\Gamma}(x)= \begin{cases}1, & x \in \Gamma \\ 0, & x \bar{\in} \Gamma\end{cases}
$$

In other words, the sequence $\left\{g_{\Gamma, \lambda}(z)\right\}$ converges uniformly to the analytical functions $\psi(z)$ and 0 in the regions $\Gamma+i R^{4}$ and $\left(R^{4} \backslash \bar{\Gamma}\right)+i R^{4}$ correspondingly.

(II) Let a function of four variables $F(x)$ be locally integrable and

$$
\lim _{\lambda \rightarrow 0} \int d^{4} x F(x) g_{\Gamma, \lambda}(x)=0
$$

for $\forall g_{\Gamma, \lambda}(z) \in \Pi(G)$. Then

for $\forall x \in G$.

$$
F(x)=0
$$

(III) Let us consider the functional

$$
\int_{L} d^{4} z F(z) g_{\Gamma, \lambda}(z)
$$


where $L$ is a surface of integration in $C^{4}$. Let the surface of integration $L$ be deformed continuously in the region of analyticity of the function $F(z)$ to a surface $L_{1}$ such that either

or

$$
L_{1} \subset \Gamma+i R^{4}
$$

Then

$$
L_{1} \subset\left(R^{4} \backslash \bar{\Gamma}\right)+i R^{4} \text {. }
$$

$$
\begin{aligned}
& \lim _{\lambda \rightarrow 0} \int_{L_{1}} d^{4} z F(z) g_{\Gamma, \lambda}(z)=\int_{L_{1}} d^{4} z F(z) \lim _{\lambda \rightarrow 0} g_{\Gamma, \lambda}(z) \\
& = \begin{cases}\int d_{L_{1}} d^{4} F(z) \psi(z), & L_{1} \subset \Gamma+i R^{4} ; \\
0 \quad, & L_{1} \subset\left(R^{4} \backslash \bar{\Gamma}\right)+i R^{4} .\end{cases}
\end{aligned}
$$

\section{The Principle of Physical Locality}

As is well known [1], when constructing the perturbation series for the $S$-matrix in local quantum field theory one finds that the expression

$$
S_{n}\left(x_{1}, \ldots, x_{n}\right)=T\left(\mathscr{L}_{I}\left(x_{1}\right) \ldots \mathscr{L}_{I}\left(x_{n}\right)\right)
$$

is not the most general one that satisfies the requirements of Lorentz covariance, unitarity and causality. These conditions completely determine $S_{n}\left(x_{1}, \ldots, x_{n}\right)$ up to a certain quasilocal operator $A_{n}\left(x_{1}, \ldots, x_{n}\right)$, the coefficient functions of which have the form

$$
B\left(\cdots \frac{\partial}{\partial x_{j}} \cdots\right) \delta^{(4)}\left(x_{1}-x_{2}\right) \ldots \delta^{(4)}\left(x_{1}-x_{n}\right) .
$$

Here $B$ is independent of $x_{j}$ due to considerations of translation invariance and contains derivatives of a finite order only.

This conclusion is based, firstly, on the causality condition which completely determines the operator function $S_{n}\left(x_{1}, \ldots, x_{n}\right)$ in terms of the preceding functions $S_{1}, S_{2}, \ldots, S_{n-1}$ within the domain of definition of its arguments in which $x_{1} \gtrsim x_{j}$ (for at least one of $j=2,3, \ldots n$ ) and, secondly, on the postulate that the coefficient functions of the operator $S_{n}\left(x_{1}, \ldots, x_{n}\right)$ in (5.2) are tempered distributions.

Thus the causality condition (3.1) in the Bogolubov and Shirkov [1] formulation gives rise to the requirement for the quasilocal operator $\Lambda_{n}\left(x_{1}, \ldots, x_{n}\right)$ to be located at the point $x_{1}=x_{2}=\cdots=x_{n}$. This requirement guarantees undoubtedly the fulfilment of the causality condition but it is too strong and can be weakened. How is it possible to do this?

The action of the local and quasilocal distributions is usually thought of as follows: 
Definition 1. The Lorentz invariant distribution $K\left(x_{1}-x_{2}\right)$ is called local and located at the point $x_{1}=x_{2}$ if

$$
\int d^{4} x_{1} \int d^{4} x_{2} f_{G_{1}}\left(x_{1}\right) K\left(x_{1}-x_{2}\right) f_{G_{2}}\left(x_{2}\right)=0
$$

for

$$
\forall f_{G_{j}}(x) \in D\left(G_{j}\right) \quad(j=1,2)
$$

if

$$
G_{1} \cap G_{2}=\varnothing .
$$

Here $D(G)$ is the space of infinitely differentiable functions with a finite support located in $G$.

The general form of the local distribution satisfying the Definition 1 is

$$
\text { a) } K\left(x_{1}-x_{2}\right)=\sum_{n=0}^{N} C_{n} \square^{n} \delta^{(4)}\left(x_{1}-x_{2}\right)
$$

for a certain $N<\infty$ in the case of tempered distributions and

b) $K\left(x_{1}-x_{2}\right)=\sum_{n=0}^{\infty} C_{n} \square^{n} \delta^{(4)}\left(x_{1}-x_{2}\right)$

in the case of the Jaffe space $[8,9]$ if

where

$$
\int_{0}^{\infty} \frac{d u \log ^{+}\left|K\left(u^{2}\right)\right|}{1+u^{2}}<\infty
$$

$$
\left|K\left(u^{2}\right)\right|=\sum_{n=0}^{\infty}\left|C_{n}\right| u^{2 n} .
$$

As was noted above, the use of these local functions or distributions for constructing the quasilocal operators guarantees the validity of the microcausality condition (3.1) or (3.2). However, from the point of view of causality our restriction to considering the distributions located at a point seems to be too strong. In fact, when verifying causality and local commutativity conditions or studying propagation of wave packets we have to consider only the regions separated by space-like surfaces rather than any space-time regions, for we always wonder how one or another event develops in time.

Thus, the problem of the causal influence of one space-time region on another can be formulated correctly if and only if the both regions are separated by a space-like surface. Otherwise in each region there will exist points located in the causal cones of some other region, and the events occuring in these regions can mutually affect one another. Therefore it is impossible to see how changes of the events in one region affect the changes in another. Thus we are led to

Definition 2 (the principle of physical locality). 
The Lorentz invariant distribution $K\left(x_{1}-x_{2}\right)$ is called physically local, or satisfies the principle of physical locality if

$$
\begin{gathered}
\iint d^{4} x_{1} d^{4} x_{2} f_{G_{1}}\left(x_{1}\right) K\left(x_{1}-x_{2}\right) f_{G_{2}}\left(x_{2}\right)=0 \\
\forall f_{G_{j}}(x) \in D\left(G_{j}\right) \quad(j=1,2)
\end{gathered}
$$

for any regions $G_{1}$ and $G_{2}$ between which a space-like surface can be located.

It is easy to show that in the case of the tempered distributions and the Jaffe strict locality the Definition 2 is equivalent to the Definition 1, i.e. the local distributions obeying Definition 2 and invariant under Lorentz transformations are given in the general form by (5.3) and (5.4). Therefore the Definition 2 gives nothing new. However in the case of the distributions defined on the spaces of analytical functions there arise new nontrivial distributions different from (5.2)-(5.4) which obey the principle of physical locality.

Let us consider the space of test functions $Z$. In this space there is no functions with finite supports as far as it consists of entire analytical functions. Therefore we have to suggest a method with the help of which we shall investigate the space-time properties of analytical functionals in the real space $R^{4}$.

Our definition is that the space-time properties of functionals on $Z$ in the real $x$-space $R^{4}$ are determined by means of projecting sequences. Therefore the Definition 2 should be formulated in the following way.

Definition 2'. The Lorentz invariant distribution $K\left(x_{1}-x_{2}\right)$ defined on $Z$ is called physically local or satisfies the principle of physical locality if

$$
\begin{gathered}
\lim _{\lambda_{1} \rightarrow \infty} \lim _{\lambda_{2} \rightarrow \infty} \int d^{4} x_{1} \int d^{4} x_{2} f_{G_{1}, \lambda_{1}}\left(x_{1}\right) K\left(x_{1}-x_{2}\right) f_{G_{2}, \lambda_{2}}\left(x_{2}\right)=0 \\
\forall f_{G_{j}, \lambda_{j}}(x) \in \Pi\left(G_{j}\right) \quad(j=1,2)
\end{gathered}
$$

for any regions $G_{1}$ and $G_{2}$ between which a space-like surface can be located.

It is more convenient to use the Definition $2^{\prime}$ in a somewhat different form:

Definition $2^{\prime \prime}$. The Lorentz invariant distribution $K(x)$ defined on $Z$ is called physically local if

$$
\begin{gathered}
\lim _{\lambda \rightarrow 0} \int d^{4} x K(x) g_{\Gamma, \lambda}(x)=0, \\
\forall g_{\Gamma, \lambda}(x) \in \Pi(G) .
\end{gathered}
$$

In this case the region $G$ is such that there always exists a possibility of locating through the point $x=0$ a space-like surface which does not cross the region $G$. 


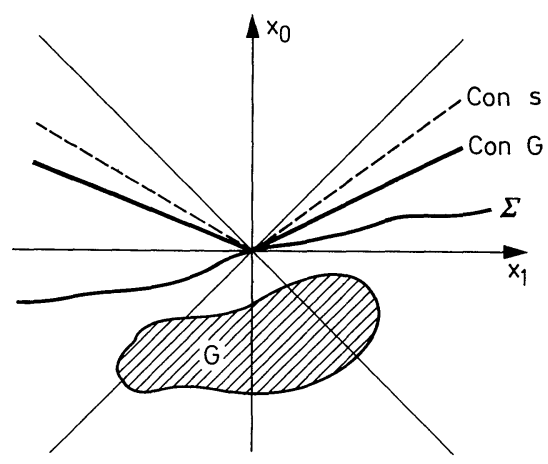

Fig. 1

It should be noted that all local distributions (5.3) and (5.4) satisfy the principle of physical locality. Therefore the investigation of the spacetime properties of the local quantum field theory with the help of projecting sequences leads to the usual results.

Now we show that the distributions $K\left(x_{1}-x_{2}\right)$ obeying the conditions (E) are physically local.

Let us consider the functional $\left(K, g_{\Gamma, \lambda}\right)$. Making use of $(2.5)$ we can obtain

$$
\begin{aligned}
\left(K, g_{\Gamma, \lambda}\right) & =\int d^{4} x K(x) g_{\Gamma, \lambda}(x) \\
& =\int_{\varrho_{0}^{2}+\varrho^{2} \leqq l^{2}} d^{4} \varrho a\left(\varrho^{2}\right) g_{\Gamma, \lambda}\left(i \varrho_{0}, \varrho\right)
\end{aligned}
$$

where $g_{\Gamma, \lambda}(z) \in \Pi(G)$. Let $\Sigma$ be a space-like surface satisfying the conditions of the Definition 2".

Without loss of generality the region $G$ may be considered to be in the past with respect to the surface $\Sigma$ as is shown in Fig. 1. Since the region $G$ is bounded there always exists such a space-like cone

$$
\operatorname{Con}_{G}=\left\{x: x_{0} \geqq 0, x_{0}^{2}=A_{G}^{2} x^{2} ; 0<A_{G}<1-\gamma, \gamma>0\right\}
$$

that the region $G$ lies in the past with respect to this cone. The constant $A_{G}$ is strictly smaller than unity and depends on the region $G$.

Let us rewrite the integral (5.7) in spherical coordinates

$$
\begin{aligned}
& \left(K, g_{\Gamma, \lambda}\right)=\int_{0}^{l} d \varrho \varrho^{3} \int_{0}^{\pi} d \alpha \operatorname{Sin}^{2} \alpha \int_{0}^{\pi} d \theta \operatorname{Sin} \theta \int_{0}^{2 \pi} d \varphi a\left(\varrho^{2}\right) \\
& \cdot g_{\Gamma, \lambda}(i \varrho \operatorname{Cos} \alpha, \varrho \operatorname{Sin} \alpha \operatorname{Cos} \theta, \varrho \operatorname{Sin} \alpha \operatorname{Sin} \theta \operatorname{Cos} \varphi, \varrho \operatorname{Sin} \alpha \operatorname{Sin} \theta \operatorname{Sin} \varphi) .
\end{aligned}
$$

The integration over $\alpha$ is carried out along the segment $[0, \pi]$ in the plane of the complex variable $\alpha+i \beta$. Because the integrand is an entire 


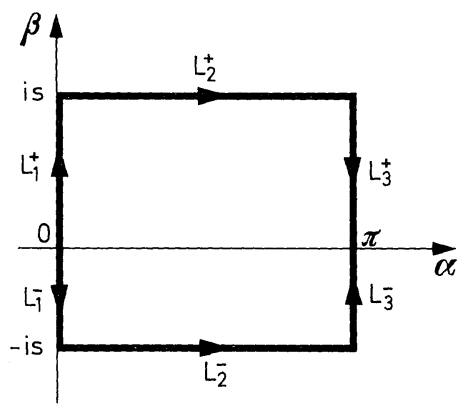

Fig. 2

function when $\lambda>0$ the contour of integration can be displaced as is shown in Fig. 2. Here $s$ is an arbitrary positive number. It is easy to verify that the real parts of the arguments $z_{j}(j=0,1,2,3)$ of the function $g_{\Gamma, \lambda}\left(z_{0}, z_{1}, z_{2}, z_{3}\right)$ in (5.8) behave like:

(1) on the contours $L_{1}^{+}=[0, i s]$ and $L_{3}^{+}=[\pi+i s, \pi]$

$$
\operatorname{Re} z_{j}=x_{j}=0 \quad(j=0,1,2,3) ;
$$

(2) on the contour $L_{2}^{+}=[i s, i s+\pi]$

$$
\begin{aligned}
& x_{0}=\varrho \mathrm{Sh} s \cdot \operatorname{Sin} \alpha \\
& x_{1}=\varrho \mathrm{Ch} s \cdot \operatorname{Sin} \alpha \operatorname{Cos} \theta \\
& x_{2}=\varrho \mathrm{Ch} s \cdot \operatorname{Sin} \alpha \operatorname{Sin} \theta \operatorname{Cos} \varphi \\
& x_{3}=\varrho \mathrm{Ch} s \cdot \operatorname{Sin} \alpha \operatorname{Sin} \theta \operatorname{Sin} \varphi
\end{aligned}
$$

i.e. these points are on the cone

$$
\mathrm{Con}_{s}=\left\{x: x_{0} \geqq 0, x_{0}^{2}=(\text { th } s)^{2} x^{2}\right\} .
$$

If $s$ is chosen large enough so that

$$
A_{G}<\text { th } s<1,
$$

which can always be done, $\mathrm{Con}_{s}$ lies inside $\mathrm{Con}_{G}$ (see Fig. 1).

Thus, the integration region is outside $G+i R^{4}$ and on it any projecting sequence from $\Pi(G)$ converges uniformly to zero. Therefore the condition (5.6) is valid.

If $G$ lies in the future with respect to the surface $\Sigma$ then to prove (5.6) it is necessary to pass in (5.8) to an integration over the contours $L^{-}=\left\{L_{1}^{-}=[0,-i s], L_{2}^{-}=[-i s, \pi-i s], L_{3}^{-}=[\pi-i s, \pi]\right\}$ (see Fig. 2).

Finally the distributions satisfying the conditions $(E)$ are physically local but are not local because they do not obey the Definition 1 . 


\section{Causality of Retarded Functions}

In order to demonstrate the mechanism of the fulfilment of the causality condition we consider a nonlocal retarded function $D_{\text {ret }}(x)$ the Fourier transform of which has the form

$$
\tilde{D}_{\mathrm{ret}}(p)=\frac{W\left(p^{2}\right)}{m^{2}-p^{2}-i p_{0} \varepsilon} .
$$

Here $W\left(p^{2}\right)$ satisfies the conditions $(E)$.

The function $D_{\text {ret }}(x)$ is a distribution defined on the space $Z$. The following representation is valid:

where

$$
\left(D_{\mathrm{ret}}, f\right)=\int_{\varrho^{2} \leqq l^{2}} d^{4} \varrho a\left(\varrho^{2}\right) \int d^{4} x \Delta_{\mathrm{ret}}\left(m, x^{2}\right) f\left(x_{0}+i \varrho_{0}, \boldsymbol{x}+\varrho\right)
$$

$\Delta_{\text {ret }}\left(m, x^{2}\right)=\frac{\Theta\left(x_{0}\right)}{2 \pi}\left\{\delta\left(x^{2}\right)-\Theta\left(x^{2}\right) \frac{m}{2 \sqrt{x^{2}}} J_{1}\left(m \sqrt{x^{2}}\right)\right\}, \quad x^{2}=x_{0}^{2}-\boldsymbol{x}^{2}$.

Our problem is to prove that

$$
\lim _{\lambda \rightarrow 0}\left(D_{\text {ret }}, g_{\Gamma, \lambda}\right)=0
$$

for any projecting sequences $g_{\Gamma, \lambda} \in \Pi(G)$ if $G \bar{C} V^{+}$.

As in the previous section the proof consists in the following. In the representation (6.2) the surface of integration can be chosen for any bounded $G$ outside the future cone $V^{+}$in such a way that any projecting sequence from $\Pi(G)$ converges uniformly to zero.

Thus, we consider

$$
\begin{aligned}
\left(D_{\text {ret }}, g_{\Gamma, \lambda}\right) & =\int_{\varrho^{2} \leqq l^{2}} d^{4} \varrho a\left(\varrho^{2}\right) f_{\Gamma^{-}, \lambda}\left(i \varrho_{0}, \varrho\right) \\
f_{\Gamma^{-}, \lambda}(z) & =\int d^{4} x^{\prime} \Delta_{\mathrm{ret}}\left(m, x^{\prime 2}\right) g_{\Gamma, \lambda}\left(z+x^{\prime}\right) .
\end{aligned}
$$

The functions $f_{\Gamma^{-}, \lambda}(z)$ converge uniformly to zero outside the region

$$
\begin{aligned}
G^{-} & =\left\{z: x=\xi+\eta, \xi \in G, \eta \in V^{-} ; y \in R^{4}\right\} \\
V^{-} & =\left\{x: x_{0}<0, x^{2}>0\right\}
\end{aligned}
$$

for all $\Gamma \subset G$. For any bounded $G \bar{C} V^{+}$there exists such a cone

$$
\operatorname{Con}_{G}=\left\{x: x_{0} \geqq 0, x_{0}^{2}=A_{G}^{2} x^{2}, 0<A_{G}<1-\gamma, \gamma>0\right\}
$$

that the region $G^{-}$and, consequently, any $\Gamma^{-} C G^{-}$lies outside this cone. As far as the cone $\mathrm{Con}_{G}$ is a space-like surface with respect to which the region $G^{-}$lies in the past all the statements of the Section 5 
are valid and, consequently, the condition (6.4) is valid too. This means that the retarded function (6.1) satisfies the microcausality condition.

Now let us consider the general case. Let a retarded function $D_{\text {ret }}(x)$ allows the Jost-Lehman-Dyson representation [12] in the momentum space:

$\tilde{D}_{\mathrm{ret}}(p)=\int d^{4} u\left\{\int_{M^{2}}^{\infty} \frac{d m^{2} W_{1}\left((u-p)^{2}\right) \phi\left(u, m^{2}\right)}{W_{1}\left(m^{2}\right)\left(m^{2}-(p-u)^{2}-i \varepsilon(p-u)_{0}\right)}+W_{2}\left(u,(u-p)^{2}\right)\right\}$.

It is assumed that all integrals in this representation converge well. The function $W_{1}(z)$ satisfies the conditions $(E)$ and have no zeros for $\operatorname{Re} z>0$. The function $W_{2}(u, z)$ satisfies the conditions $(E)$ with respect to the second variable $z$ uniformly relative to the first variable $u$. The integration over $u$ is performed over a bounded region. The functional (6.7) is a distribution defined on $Z$, i.e. the functional

$$
\left(D_{\text {ret }}, f\right)=\int d^{4} p \tilde{D}_{\text {ret }}(p) \tilde{f}(p)
$$

exists. After simple manipulations one obtains easily

$\left(D_{\text {ret }}, f\right)=\int d^{4} u \underset{\varrho^{2} \leqq l^{2}}{\int} d^{4} \varrho \int d^{4} x f\left(x_{0}+i \varrho_{0}, \boldsymbol{x}+\varrho\right)$
$\cdot e^{-i\left[u_{0}\left(x_{0}+i \varrho_{0}\right)-\boldsymbol{u}(\boldsymbol{x}+\varrho)\right]} \cdot\left\{a_{1}\left(\varrho^{2}\right) \int_{M^{2}}^{\infty} \frac{d m^{2} \phi\left(u, m^{2}\right)}{W_{1}\left(m^{2}\right)} A\left(m, x^{2}\right)+\delta^{(4)}(x) a_{2}\left(u, \varrho^{2}\right)\right\}$.

It is easy to see that the structure of this expression comes to (6.5) and (5.7). As far as we suppose that all integrations in (6.8) converge well the calculations performed above are valid in this case too. Thus we obtain

$$
\begin{gathered}
\lim _{\lambda \rightarrow 0}\left(D_{\text {ret }}, g_{\Gamma, \lambda}\right)=0 \\
\forall g_{\Gamma, \lambda} \in \Pi(G) \quad \text { if } \quad G \bar{C} V^{+} .
\end{gathered}
$$

Now the problem is to prove that in the nonlocal theory the $S$-matrix elements responsible for the fulfilment of causality permit the representation (6.7).

\section{The Representation of a Retarded Product in the $\boldsymbol{n}$-th Perturbation Order}

The retarded function $M(x)$ in (3.2) can be represented in the form

$$
\begin{aligned}
M(x) & =\sum_{n} g^{n} M_{n}(x), \\
M_{n}(x) & =\sum_{D_{n}} M_{D_{n}}(x) .
\end{aligned}
$$


In (7.2) the summation is performed over all connected diagrams $D_{n}$ in the $n$-th perturbation order.

Let us show that the representation (6.7) is valid for any matrix elements $M_{D_{n}}(x)=\left\langle\alpha\left|R_{D_{n}}(x, 0)\right| \beta\right\rangle$, i.e.

$M_{D_{n}}(x)=\int d^{4} p e^{-i p x} \tilde{M}_{D_{n}}(p)$

$\tilde{M}_{D_{n}}(p)=\int d^{4} u\left\{\int_{M^{2}}^{\infty} \frac{d m^{2} W_{1}\left((u-p)^{2}\right) \phi\left(u, m^{2}\right)}{W_{1}\left(m^{2}\right)\left(m^{2}-(p-u)^{2}-i \varepsilon(p-u)_{0}\right)}+W_{2}\left(u,(u-p)^{2}\right)\right\}$.

Here $W_{1}(z)$ and $W_{2}(u, z)$ satisfy the conditions $(E)$ and

$$
\begin{aligned}
& W(z)=O\left(\exp L_{n} \operatorname{Re} \sqrt{z}\right), \quad|z| \rightarrow \infty ; \\
& W(z)=O\left(\frac{1}{|z|}\right), \quad z \rightarrow-\infty
\end{aligned}
$$

where $L_{n}$ is a constant which depends on $l$ and the perturbation order.

The proof follows from the following statements (see [5-8]).

1. The function $\tilde{M}_{D_{n}}(p)$ is the limit of the regularized expression $\tilde{M}_{D_{n}}^{\delta}(p)$, i.e.

$$
\lim _{\delta \rightarrow 0} \tilde{M}_{D_{n}}^{\delta}(p)=\tilde{M}_{D_{n}}(p) .
$$

This limit exists, as was shown in [7].

2. The analytical properties of the limiting amplitudes $\tilde{M}_{D_{n}}(p)$ are the same as in local theory (see [5], [7]) in any finite domain of the complex momentum plane for the exception of the infinity.

3. $\tilde{M}_{D_{n}}(p)=O\left(\frac{1}{\left|p^{2}\right|}\right), \quad p^{2} \rightarrow-\infty$.

4. $\tilde{M}_{D_{n}}(p)=O\left(\exp \left[(n-1)(l+\varepsilon) \operatorname{Re} \sqrt{p^{2}}\right]\right), \quad\left|p^{2}\right| \rightarrow \infty$, $-\pi<\arg p^{2}<\pi$.

5. The $S^{\delta}$-matrix for $\delta>0$ corresponds to the local theory (see $[7,8]$ ) so that

if $x \bar{\in} V^{+}$.

$$
M_{D_{n}}^{\delta}(x)=0
$$

6. $\tilde{M}_{D_{n}}^{\delta}(p)=O\left(\frac{1}{\left|p^{2}\right|^{2}}\right)$ for $\left|p^{2}\right| \rightarrow \infty$ and $\delta>0$.

Making use of the Properties 5 and 6 and following papers $[12,13]$ one obtains the representation

$$
\tilde{M}_{D_{n}}^{\delta}(p)=\int d^{4} u \int_{0}^{\infty} \frac{d \varkappa^{2} \phi^{\delta}\left(u, \varkappa^{2}\right)}{\left[\varkappa^{2}-(p-u)^{2}\right]_{\mathrm{ret}}}
$$


where the function $\phi^{\delta}\left(u, \varkappa^{2}\right)$ vanishes outside the region

$$
S=\left\{\begin{array}{l}
\left(\frac{Q+K}{2}-u\right) \in V^{+} \\
\left(\frac{Q+K}{2}+u\right) \in V^{+} \\
x^{2}=\max \left\{0, m-\sqrt{\left(\frac{Q+K}{2}-u\right)^{2}}, m-\sqrt{\left(\frac{Q+K}{2}+u\right)^{2}}\right\}
\end{array}\right.
$$

Here $Q$ and $K$ are the momenta of states $|\alpha\rangle$ and $|\beta\rangle$. The analytic structure of $\tilde{M}_{D_{n}}^{\delta}(p)$ with respect to $p$ is singled out explicitly in the representation $(7.5)$.

The Property 2 requires that

$$
\begin{aligned}
\lim _{\delta \rightarrow 0} \tilde{M}_{D_{n}}^{\delta}(p)= & M_{D_{n}}(p) \\
= & \int d^{4} u\left\{\int_{0}^{\infty} \frac{d \varkappa^{2} W_{1}\left((u-p)^{2}\right) \phi\left(u, \varkappa^{2}\right)}{W_{1}\left(\varkappa^{2}\right)\left(\varkappa^{2}-(p-u)^{2}\right)_{\mathrm{ret}}}\right. \\
& \left.+W_{2}\left(u,(p-u)^{2}\right)\right\}
\end{aligned}
$$

where $W_{1}(z)$ and $W_{2}(u, z)$ are entire functions of $z$, and the integrals in (7.6) converge.

From the Properties 3 and 4 it follows that the order of growth of $W_{1}(z)$ and $W_{2}(u, z)$ is $\varrho=\frac{1}{2}$ and for $z \rightarrow-\infty$

$$
\begin{aligned}
& W_{1}(z) \rightarrow 0 \\
& W_{2}(u, z) \rightarrow 0 \quad \forall u \in S .
\end{aligned}
$$

From the Property 1 it follows

and

$$
\Phi\left(u, x^{2}\right)=\lim _{\delta \rightarrow 0} \Phi^{\delta}\left(u, \varkappa^{2}\right)
$$

$$
\Phi\left(u, \varkappa^{2}\right)=O\left(e^{(n-1)(l+\varepsilon) \varkappa}\right) \quad x \rightarrow \infty .
$$

The representation (7.6) can be obtained from (7.5) with the help of the transition to the limit $\delta \rightarrow 0$. In order to perform this transition we should write down a certain representation for $\tilde{M}_{D_{n}}^{\delta}(p)$ equivalent to (7.5) which permits the transition to the limit $\delta \rightarrow 0$ in the integrand. According to the Property 1 , the limiting expression is just the described representation for $\tilde{M}_{D_{n}}(p)$.

As far as the representation (7.6) is valid for any connected diagram in perturbation theory the condition of microcausality (3.2) turns out to be valid in the $n$-th perturbation order. 


\section{Conclusion}

Thus it turned out that the nonlocal $S$-matrix constructed with the help of entire analytical functions of the order $1 / 2$ satisfies the condition of microcausality. Now it is still difficult to estimate the value of this fact and understand more deeply the reasons underlying this result.

It is quite possible that we have a generalization of the Schrödinger equation of the following type. In the local case we have

where

$$
i \frac{\partial}{\partial t} \psi(t)=g H_{I}(t) \psi(t)
$$

$$
g H_{I}(t)=g \int d \boldsymbol{x}: \varphi^{N}(\boldsymbol{x}, t):
$$

This equation leads formally to the microcausal $S$-matrix at least because the interaction is local.

Formally our introduction of nonlocality can be represented as

i.e.

$$
\varphi(\boldsymbol{x}, t) \rightarrow \int_{\varrho^{2} \leqq l^{2}} d^{4} \varrho a\left(\varrho^{2}\right) \varphi\left(\boldsymbol{x}+\varrho, t+i \varrho_{0}\right)
$$

$$
H_{I}(t) \rightarrow \int_{-l}^{l} d \tau H_{I}(t+i \tau) .
$$

It should be noted that these formulas are completely formal because there are no any operator realization of nonlocal theory without using a regularization procedure. Though the Hamiltonian (8.1) corresponds to the nonlocal theory the solution of the equation

$$
i \frac{\partial}{\partial t} \psi(t)=g \int_{-l}^{l} d \tau H_{I}(t+i \tau) \psi(t)
$$

may have the main properties of the microcausal theory.

These problems deserve further study.

Acknowledgments. The authors wish to thank Professors D. I. Blokhintsev, B. M. Barbashov, N. A. Chernikov, and V. Ja. Fainberg for useful discussions.

\section{References}

1. Bogolubov, N.N., Shirkov,D.V.: Introduction to the theory of quantized fields. New York: Interscience 1959

2. Streater,R.F., Wightman,A.S.: PCT, spin and statistics and all that. New YorkAmsterdam: W. A. Benjamin, Inc. 1964

3. Bogolubov, N.N., Medvedev,B.V., Polivanov,M.K.: Problems in the theory of dispersion relations. Institute for Advanced Studies, Princeton, 1958 
4. Blokhintsev, D. I.: Space and time in the microworld. Dordrecht: D. Reidel, Publishing Company, 1973

Wanders, G.: Nuovo Cim. 14, 168 (1959)

Eden, R. J., Landshoff, P.V.: Ann. Phys. 31, 370 (1965)

Stapp, H. P.: Phys. Rev. 139, 257 (1965)

Kirznits, D. A.: Proceedings of the I. International Seminar on Nonlocal Quantum Field Theory, JINR, P2-3590, Dubna, 1967

5. Efimov, G. V.: Preprints ITF, Kiev, N. 52, 54, 55 (1968)

Problems of Physics, E. Ch. A. Ja. 1, 1, Atomizdat, 1970

Proceedings of the II. and III. International Seminars on Nonlocal Quantum Field Theory, JINR, 2-5400, Dubna, 1970

D2-7161, Dubna, 1973

6. Efimov, G.V.: Preprint ITF-68-52, Kiev, 1968, Commun. math. Phys. 7, 138, 1968

7. Alebastrov, V.A., Efimov, G.V.: JINR P2-6586, Dubna, 1972, Commun. math. Phys. 31, 1 (1973)

8. Efimov, G.V.: JINR P2-6864, Dubna, 1972

9. Jaffe, A. M.: Phys. Rev. Letters 17, 661 (1966); Phys. Rev. 158, 1454 (1967)

10. Jost, R.: The general theory of quantized fields. Lectures in Applied Mathematics, American Mathematical Society, Providence, Rhode Island, 1965

11. Efimov, G. V.: JINR, P2-6756, Dubna, 1972

12. Schweber, S.: An introduction to relativistic quantum field theory, part one. New York: Harper and Row, 1961

Dyson,F. J.: Phys. Rev. 110, 1460 (1958)

Jost, R., Lehmann, H.: Nuovo Cim. 5, 1598 (1957)

13. Fainberg, V. J.: JETP (sov.) 36, 1503 (1959)

Communicated by K. Hepp

\author{
V. A. Alebastrov \\ G. V. Efimov \\ Joint Institute for Nuclear Research \\ Laboratory of Theoretical Physics \\ Head Post Office \\ P. O. Box 79 \\ Moscow, USSR
}

